\title{
An Optimized Collision Avoidance Decision-Making System for Autonomous Ships under Human-Machine Cooperation Situations
}

\author{
Xiaolie Wu $\mathbb{D}^{\mathbb{D}},{ }^{1}$ Kezhong Liu, ${ }^{1,2}$ Jinfen Zhang, ${ }^{3}$ Zhitao Yuan, ${ }^{1,2}$ Jiongjiong Liu, ${ }^{3}$ and Qing Yu $\mathbb{D}^{1}$ \\ ${ }^{1}$ School of Navigation, Wuhan University of Technology, Wuhan 430063, China \\ ${ }^{2}$ Hubei Key Laboratory of Inland Shipping Technology, Wuhan 430063, China \\ ${ }^{3}$ National Engineering Research Center for Water Transport Safety, Wuhan University of Technology, Wuhan 430070, China
}

Correspondence should be addressed to Qing Yu; qing.yu@whut.edu.cn

Received 13 July 2021; Accepted 18 August 2021; Published 27 August 2021

Academic Editor: Xinqiang Chen

Copyright (c) 2021 Xiaolie Wu et al. This is an open access article distributed under the Creative Commons Attribution License, which permits unrestricted use, distribution, and reproduction in any medium, provided the original work is properly cited.

Maritime Autonomous Surface Ships (MASSs) are attracting increasing attention in recent years as it brings new opportunities for water transportation. Previous studies aim to propose fully autonomous system on collision avoidance decisions and operations, either focus on supporting conflict detection or providing with collision avoidance decisions. However, the human-machine cooperation is essential in practice at the first stage of automation. An optimized collision avoidance decision-making system is proposed in this paper, which involves risk appetite (RA) as the orientation. The RA oriented collision avoidance decision-making system (RA-CADMS) is developed based on human-machine interaction during ship collision avoidance, while being consistent with the International Regulations for Preventing Collisions at Sea (COLREGS) and Ordinary Practice of Seamen (OPS). It facilitates automatic collision avoidance and safeguards the MASS remote control. Moreover, the proposed RA-CADMS are used in several encounter situations to demonstrate the preference. The results show that the RA-CADMS is capable of providing accurate collision avoidance decisions, while ensuring efficiency of MASS maneuvering under different RA.

\section{Introduction}

The idea of embracing autonomous vessels to create sustainable development and shape new opportunities for water transportations in the industry is getting feasible. As the technological barriers are resolved in numerous studies, autonomous vessels have been drawing significant attention recently. For autonomous vessels, the collision avoidance system (CAS) and the control system are two fundamental systems, which are closely related with vessel maneuvering and safety performance. Both of them aim at removing human operator in the control loop, which is still questionable as teaching the automation to understand regulations is still unrealistic and the trust of public on the autonomous vessels is challenging [1]. However, human intelligence and machine intelligence are complementary; the former one is good at experience and the later one shows high power of computing
[2]. Therefore, the human-machine cooperation is essential for developing automatic system for the Maritime Autonomous Surface Ships (MASS) [3].

Several studies tried to propose intelligent collision avoidance and ship control systems to support autonomous vessels navigating in complex waters. However, the majority of them are purely based on geometrical collision avoidance approaches (e.g., CPA approach, fuzzy logic [4], artificial potential field method [5], velocity obstacle [6,7], model predictive control (MPC) [8], and Convention on the International Regulations for Preventing Collisions at Sea (COLREGS), ignoring the valuable experience from crewmembers in practices, leading to unreasonable decision making for the autonomous vessel in real encounter situations. This question has been highlighted in the first generation of autonomous vessels, in which the unmanned and manned vessels exist when the response of autonomous 
vessels to collision risk is still uncertain [9]. Automation can improve vessel safety from some perspectives by removing the crews while creating other unpredictable risks.

In the real practice, the collision avoidance decisions for the manned vessels are made by the officers who are on watching (OOWs). Although these decisions should be consistent with COLREGS, the OOWs will make different choices based on their risk appetite (RA). Several related factors should be considered by the OOWs, such as the water condition, ship conditions, and weather conditions. Therefore, to develop an intelligent CAS for autonomous vessels, the OOW' experience of RA and maneuvering behaviors should be fully considered to make the proposed system more practical and comprehensible under manmachine symbiosis condition.

To resolve the question, the contribution of this paper is to pioneer an optimized collision avoidance decision-making system for autonomous ships in complex encounter situations, involving RA as the orientation. The RA oriented collision avoidance decision-making system (RA-CADMS) is developed based on human-machine interaction during ship collision avoidance, consistent with COLREGS and Ordinary Practice of Seamen (OPS). It is capable of providing the decisions according to the RA under different encounter situations and the operator's risk tolerance, reflecting OOWs' experience under complex water situation that lacks information, so that realizing human-machine cooperated collision avoidance decisionmaking. The study firstly analyzes the ship collision avoidance decision behavior under various ship encounter situations. Then, a multicriteria ship collision avoidance decision-making optimization system is developed to demonstrate the decision preference of OOWs who have different RA. At last, a prospect theory (PT) is applied to balance the accepted risk thresholds in the field of ship collision avoidance system. The main contribution of this paper can be highlighted as follows:

(1) A RA-CADMS is proposed to incorporate hybrid intelligence that combines the complementary strength of experience from OOWs.

(2) The balance of collision risk tolerance and avoidance decision efficiency is resolved by the RA-CADMS by pioneering $\mathrm{PT}$ in the collision avoidance decisionmaking.

(3) The decision space for the MASS in different encounter situations is visualized and can be selected with different levels of RA.

The rest of the paper is organized as follows: previous studies related to risk appetite and ship collisions are reviewed in Section 2. In Section 3, an optimized collision avoidance decision-making system is established, and several case studies are implemented in Section 4. The discussion and conclusions are drawn in Sections 5 and 6.

\section{Literature Review}

2.1. Collision Avoidance System. As defined by [10], a generalized CAS should at least contain collision risk assessment, action decision-making, and action execution modules, in which the decision selection is the core of the CAS as it is essential for safe navigation of MASS [11].

To develop a sufficient CAS to support collision avoidance, various methods are proposed in the previous studies. They are grouped into three perspectives. First, statistical analysis and numerical method are used to support collision avoidance, such as encounter situation and stage discrimination quantitative model [12], with collision parameters. Reference [13] proposed the Personifying Intelligent Decision-making for Vessel Collision Avoidance (PIDVCA) algorithm, which quantified the initial timing of steering rudder and the last time for steering rudder. On the basis of the COLREGS, Woerner et al. [14] and Chen et al. [6]. suggested a quantitative evaluation approach to analyse the collision risk. The second group covers the knowledgebased marine collision avoidance system to improve the stability and comprehensibility of the models [15]. This type of CAS selects the optimal collision avoidance scheme through various ship dynamic parameters, e.g., course [16], relative speed [10], and ship trajectories [17]. The applied approaches in the CAS include fuzzy logic [4] and Bayesian network [18]. The last group applies advanced artificial intelligence to deal with the problem and has made great progress on collision avoidance system formulation. For example, Shen et al. [19]. utilised the deep reinforcement learning method to solve the collision avoidance problem under multiship encounter situations in restricted waters; $\mathrm{Hu}$ et al. [20]. developed a multiobjective optimization model for COLREGS-compliant path planning; improved cultural particle swarm was introduced into ship collision avoidance decision [21]. To support multiple ships anticollision decision, CAS based on multiagent was designed, in which the agents formulate collision-free strategy through information interaction [22], such as distributed algorithm [23, 24] and centralized algorithm [25]. Moreover, big data processing techniques for ship AIS trajectories and video detection provided well support to identify ship encounter behaviors more accurately $[26,27]$ or study the traffic flows in water areas [28].

It can be found that the section of collision avoidance is greatly enriched by previous studies, However, most of them ignore the impacts from human that include the common practices of seafarers, good seamanship, and risk tolerance.

2.2. Collision Avoidance System for MASS. The current studies related to MASS collision avoidance can be categorized into two groups [9]. One aims to developing the alert systems for MASS to detect potential dangers of collision, using collision risk index [29-31], ship domain [32], dangerous region in velocity-space [33] and probability of collision [34], risk prediction based on deep learning [35], etc. Different from the manned ship, objects of CAS for MASS are conflict detections, which are difficult in the perception of collision situations [9]. Although the expertbased methods and model-based methods are widely presented to support the CAS design for MASS, limitations on these methods are noted: (1) there are no common 
agreements on the boundary to clarify the safety and danger; (2) the CAS hardly provide personalized alarm services for officers with different risk attitudes.

Another group concentrates on the automatic collisionfree solutions for MASS, while the interactions of the human-machine cooperation encounter situations are not considered. According to the current studies (e.g., Huang et al. [9]), there are six kinds of collision-free resolution methods for MASS, including rule-based method, virtual vector method, discretization of solutions, continuous solutions, replanning method, and hybrid method. Three objectives include (1) offering an optimal/feasible scheme; (2) safety checking on scheme; and (3) defining all the unsafe situations. However, some of the methods are difficult to be applied in unmanned situations. For instance, Johansen et al. [8] proposed a predictive control model to offer collision avoidance by considering the maneuvering ability, while it might be difficult for MASS controller to understand the effects of these forces and how to select a collision scheme. Besides, the optimal solution offered by CAS may not be accepted by the MASS controller as the risk attitude for individuals is inconsistent.

The maneuver decision preferences for manned vessel vary with several influencing factors [36-38], investigating the maneuver decision preferences in navigating ships to avoid a collision and to ensure safety for the MASS. However, relevant studies are rarely found in the current literature due to the lack of theoretical studies.

2.3. Risk Appetite in Decision-Making. RA can be defined as the amount and type of risk that an individual is willing to pursue or retain, or the willingness to take on risky activities in pursuit of values [39]. A high-risk appetite means high-risk tolerance and hopes to give large benefits and vice versa. For example, 60 officers perceived different risk scores and performed various ship maneuverings based on scenario survey experiments under the same encounter situations [37]. The prospect theory (PT) was firstly proposed by [40] and was then updated to cumulative prospect theory (CPT) [41] and the third generation prospect theory [42]. The theory combines behavioral science theory with multicriteria decisionmaking methods to reflect decision-makers' psychological characteristics and attitudes of loss and gain. As the PT has great advantages in describing the psychological behavior characteristics and loss of decision makers, it has been widely used in financial analysis, transportation, and other domains that involve multiattribute decision-making. For instance, Wang et al. [43]. considered the psychological behavior in the emergency decision-making and proposed a group emergency decision-making approach based on PT, which was capable of adjusting the importance of the model attributesbased experts' psychological behavior. Gao et al. [44]. proposed a path selection model based on a CPT; further, Hjorth and Fosgerau [45] used risk preference parameters of traveler to trade-off traveling cost and identify the sensitivity of each parameter impacting the daily cost. Moreover, a multiattribute decision-making method [46] based on PT was proposed to solve risk decision-making problems with interval probability. Specifically, Zhou et al. [47]. applied PT in route traffic analysis and studied the drivers' route choice behavior and developed a route selection model to aid traffic management.

It can be found from the review that PT is a sufficient approach to support human behavior-based risk modelling. It is suitable for developing intelligent CAS for the MASS that crewmembers' behavioral characteristics and risk preferences can be covered to support collision avoidance. Besides, the following problems need to be resolved during the collision avoidance decision-making:

(i) How can a CAS combine the wisdom of human and intelligence of machine in collision avoidance decision?

(ii) How to balance the safety and economy of collision avoidance decision and adjust to the crew individual risk attitude variation in decision-making?

\section{A Generic Framework of RA-CADMS}

An abstract representation of RA-CADMS is shown in Figure 1. It contains two subcomponents: the RA assessor and the CADM model. The RA assessor confirms the possible avoidance schemes for the current encounter situations through three RA-related features of safety, efficiency, and stationarity. The CADM model assigns decision weights to evaluate the features and then convert them into composite prospect value, which ranks the collision avoidance decisions for the MASS under individual risk attitude. Moreover, a real-time updated loop is added to link the two components. It provides with self-updating for the RA-CADMS when information for MASS and target ships is updated after the executed decision.

3.1. RA Assessor. A high-risk appetite means high-risk tolerance and hopes to give large benefits and vice versa. For example, a survey implemented by [37] invites 60 officers to make an avoidance decision under the same encounter situation. Although the states of the vessel, weather, and other conditions are the same, the selected starting point for avoidance and minimum distance of approaching for each of them are different as their RA and maneuvering behavior are different, thereby developing a RA assessor based on OOWs' OPS and COLREG is the major component in the RACADMS, in which influence factors for collision avoidance that related to RA are discussed.

3.1.1. Parameter Related to Ship Collision Avoidance. As Rule 16 of COLREG states that "Every vessel is directed to keep out of the way of another vessel shall, so far as possible, take early and substantial action to keep well clear," the giveway vessel can take action at any time within the time interval between the time to urgent situation and the time to close-quarter situation. However, there are great differences in trajectory characteristics under the same waters based on high-quality ship trajectory data from AIS [27, 48]. Further, video-based detection reflected more detailed and clear ship 


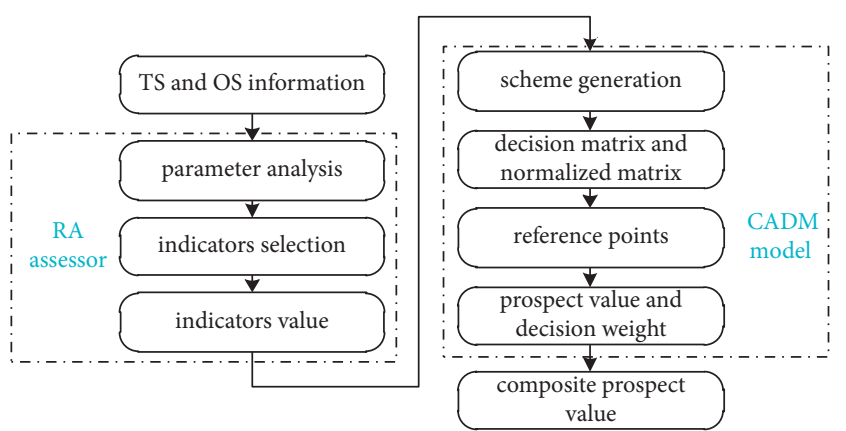

FIgURE 1: The framework for RA-CADMS.

encounter behavior, such as moving straight, turning right, and turning left, in specific waters [49]. Therefore, a high RA decision-maker may take avoidance action later than the time interval and pass the encounter at a closer distance to save fuel and time. Contrary to the high one, an OOW with low RA is cautious and prefers to take action earlier to maintain a safer distance so that leading to larger deviation and higher fuel consumptions. On such basis, three categories of RA are stated as cautious (i.e., risk aversion), aggressive (i.e., risk hobbies), and neutral (i.e., risk neutrality). Cautious refers to low risk appetite and is willing to obtain higher safety values; aggressive means higher tolerance; and neutral stands intermedium of RA.

To describe the risk of encounter situations, ship dynamic parameters are selected in the RA assessor. These include ship relative speed $\left(\mathbf{v}_{\mathrm{ij}}\right)$, accepted distance $\left(\mathbf{d}_{\mathrm{acc}}\right)$, and change of course $(\theta)$. Thus, the action time $t$ can be expressed as $t \sim\left(v_{i}, v_{j}, d_{\mathrm{acc}}, \theta, \mid P_{\mathrm{os}}, P_{\mathrm{Ts}}\right)$, where $P_{\mathrm{OS}}$ and $P_{\mathrm{TS}}$ are the positions for the own ship (OS) and the target ship
(TS). Assuming there is a MASS (i.e., ship $i$ ) encountered with a manned vessel (i.e., ship $j$ ) leads to an encounter situation of crossing over (see Figure 2). The real speeds for the ship $i$ and the ship $j$ are $v_{i}$ and $v_{j}$, respectively, and the relative speed and position between two encounters are $v_{i j}$ and $P_{i j}$. To avoid collision, the ship $i$ should take a course changing action at a certain time $t$ and position $P_{i j}(t)$ to ensure that it could pass the ship $j$ at a safety distance. If the ship $i$ takes action at time $t_{1}$ and position $P_{i j}\left(t_{1}\right)$, the minimum course change is $\theta_{1}$. Similarly, the ship $i$ should change course to $\theta_{2}$ when it gets closer (i.e., take action at time $t_{2}$ and position $P_{i j}\left(t_{2}\right)$ ).

3.1.2. Indicators for RA Assessor. Based on COLREGS, OPS, and relevant researches $[38,50,51]$, the optimization of collision avoidance decision-making for MASS is mainly reflected in safety, efficiency, and stationarity. The three indicators are proposed as follows:

(1) Safety: it is to ensure that the collision risk can be eliminated by the avoidance decisions. Although ships should pass each other not less than the minimum safety distance, the accepted distance between two ships is not persistent in real practice. It is impacted by several factors such as weather, ship type, and size and is selected based on OPS. OOWs evaluate the safety value (SV) of decision scheme mainly by the minimum passing distance $\left(d_{m}\right)$ and the time-taking evasive action to passing the close point time $\left(\mathrm{TCPA}_{a}\right)$. Thus, we define $\mathrm{SV}$ as expressed in the following equations:

$$
\begin{aligned}
& \operatorname{SV}\left(d_{m}\right)=\left\{\begin{array}{lll}
\left(\frac{d_{m}}{d_{1}}\right)^{0.65}, & d_{m} \leq d_{1}, & d_{m} \leq d_{1}, \\
1 & d_{1}<d_{m}, & d_{1}<d_{m}
\end{array}\right. \\
& \operatorname{SV}\left(\mathrm{TCPA}_{a}\right)= \begin{cases}\frac{\mathrm{TCPA}_{a}}{t_{1}}, & 0 \leq \mathrm{TCPA}_{a} \leq t_{1}, \\
1 & \mathrm{TCPA}_{a}<0 \text { or } t_{1}<\mathrm{TCPA}_{a},\end{cases} \\
& \mathrm{SV}= \begin{cases}w_{1} \mathrm{SV}\left(d_{m}\right)+w_{2} \mathrm{SV}\left(\mathrm{TCPA}_{a}\right), & d_{m} \leq D 1 \text { and } 0 \leq \mathrm{TCPA}_{a} \leq t 1, \\
1, & \text { others. }\end{cases}
\end{aligned}
$$

The thresholds $D_{1}$ and $t_{1}$ are defined to divide the safety value SV with two segments, respectively. Assuming a MASS minimum passing another TS more than $d_{1}$ or navigation at a range larger than $t_{1}$, no collision risk is existed (i.e., $\mathrm{SV}=1$ ).

(2) Efficiency: this indicator evaluates the distance deviating (Dev) of the OS from its initial course. Large Dev stands for low efficiency and small Dev means high efficiency. It can be calculated using the following equation:

$$
\operatorname{Dev}=v \times\left(t_{2}-t_{2}\right) \times \sin \theta .
$$

The Dev is influenced by the $\operatorname{OS}^{\prime}$ speed $v$, course deviation $\theta$, and the time between the course recover time $t_{2}$ and the course change time $t_{1}$. A sample is given in Figure 3. 


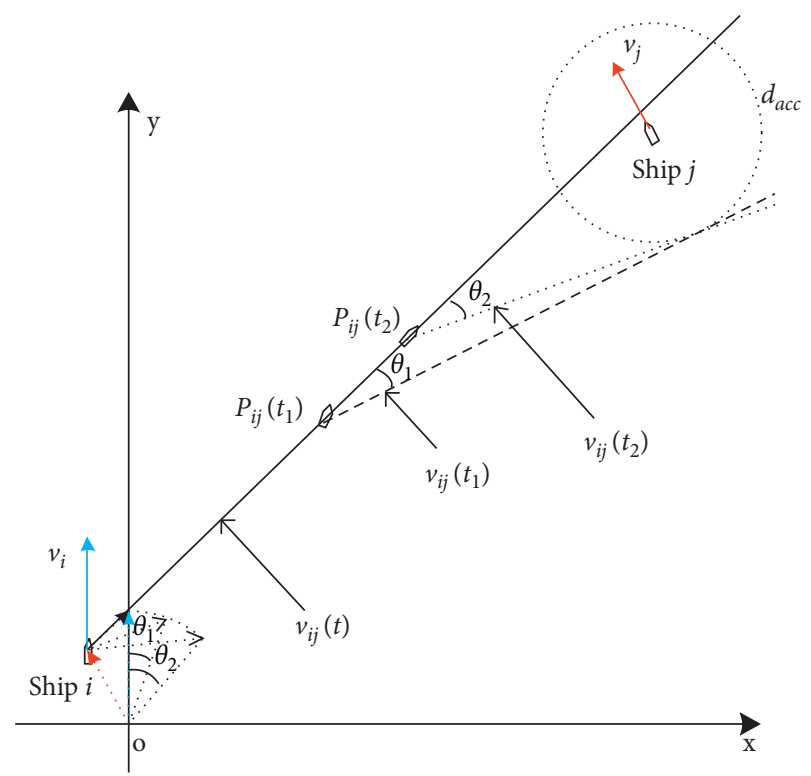

Figure 2: A sample of the autonomous vessel (own ship) encountered with a manned vessel (target ship).

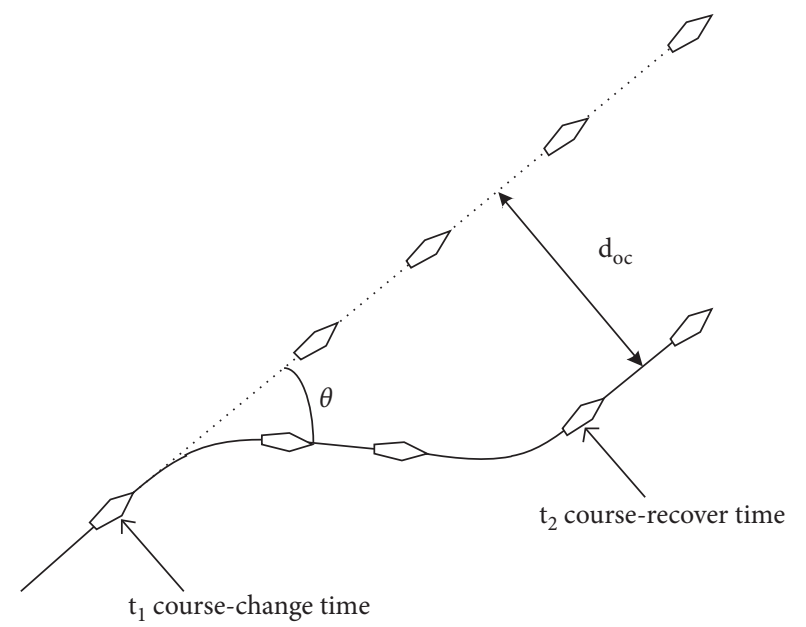

Figure 3: A sample of collision avoidance decision.

(3) Stationarity: this indicator describes the smoothness and stationarity (Sta) of the ship's trajectory in collision avoidance operation. According to Rule 18 of COLREGS, ship course and/or speed alteration should be apparent to another ship observing visually or by radar. Therefore, the course change $\theta$ of decision schemes is at least $20^{\circ}$. At the same time, considering ship maneuvering, dynamics model, and existing researches $[52,53]$, abrupt and too large course change could cause ship speed slowing down and bunker consumption. This indicator function is as follows:

$$
\text { Sta }=\pi-|\theta| \text {. }
$$

3.2. CADM Model. This section proposes a CADM model based on PT to sort the decision for different risk appetite officers. The advantage is that it takes into account the psychological characteristics through risk parameters for decision makers in the uncertainty decision progress. In the CADM model, the collision avoidance schemes are prioritized based on composite prospect values, which can be calculated through the equations introduced in the PT. As shown in Figure 4, the calculation mainly follows two steps. First, the indicator value for each scheme that selected in the RA assessor is normalized to construct the decision matrix, while the reference points for the indicators are identified by using the grey correlation analysis. Second, the prospect and objective values are aggregated by using PT, which consider the RA of the MASS and the weight of the indicators.

3.2.1. Prospect Theory. The PT was first proposed by Kahneman and Tversky [54] to explain the human behavior in a systematic way. It formulates the biased or irrational human behaviors and introduces two concepts of the definition of prospect: a value function defined on the utility and a decision weight function defined on the cumulative probability, as shown in Figure 5.

As shown in Figure 5(a), the value function is convex when the curve is in the area of gains and is concave when in the losses area. The curve in the value function is divided by the reference points $\xi_{i j}^{-}$for the positive prospect value and $\xi_{i j}^{+}$ for the negative prospect value; it is steeper for losses than for gains and can be formulated with prospect value equations.

The positive prospect value (i.e., area of gains) for $i$ th scheme and $j$ th indicator is calculated as

$$
\mathrm{pv}_{i j}^{+}=\left(1-\xi_{i j}^{-}\right)^{\alpha} \text {, }
$$

and the negative prospect value (i.e., area of losses) for $i$ th scheme and $j$ th indicator is calculated as

$$
\mathrm{pv}_{i j}^{-}=-\eta \times\left[-\left(\xi_{i j}^{+}-1\right)\right]^{\beta},
$$

where $\alpha, \beta$, and $\eta$ are the risk preference parameters. The increasing value of the parameters means the model is more sensitive on loss so that trends to avoid risk.

Figure 5(b) reports how the decision weight functions describe the well-observed behavior that human tends to overestimate the occurrence of low-probability events but underestimate that of high probability ones as

$$
\pi(\omega)= \begin{cases}\pi(\omega)^{+}=\frac{\omega^{\gamma}}{\left[\omega^{\gamma}+(1-\omega)^{\gamma}\right]^{1 / \gamma}}, & \xi_{j}^{-} \text {is reference point, } \\ \pi(\omega)^{-}=\frac{\omega^{\delta}}{\left[\omega^{\tau}+(1-\omega)^{\tau}\right]^{1 / \tau}}, & \xi_{j}^{+} \text {is reference point }\end{cases}
$$

where $\omega$ represents the objective probability of the event result; $\gamma$ and $\tau$ represent the fitting parameters of the probability weight function on the left and right sides of the reference point.

Composite prospect value of each scheme is defined as follows: 


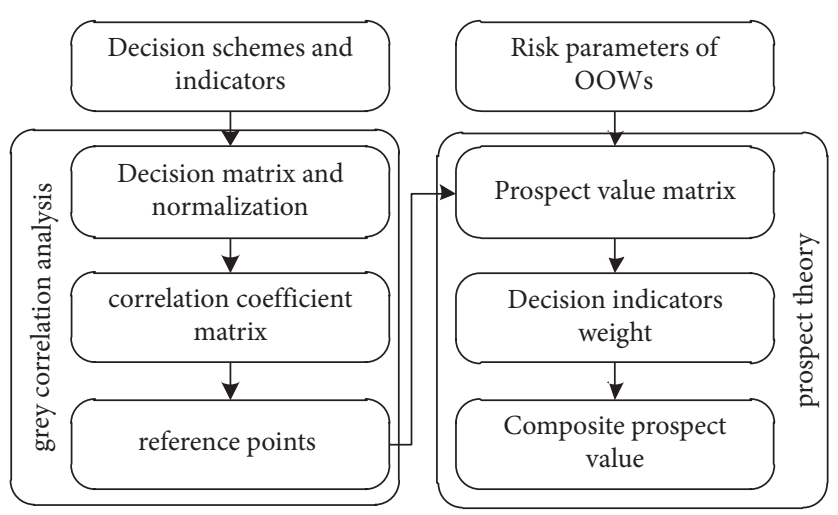

FIgURE 4: The framework for CMDA model.

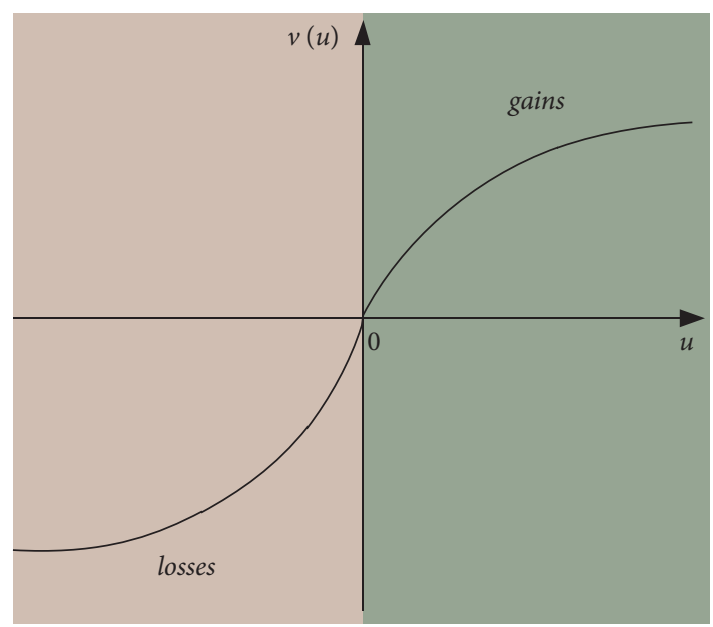

(a)

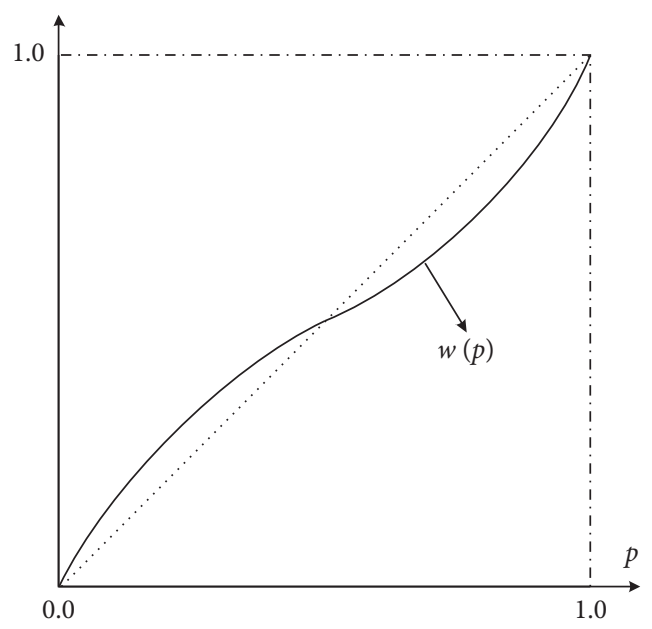

(b)

Figure 5: An example of (a) the value function and (b) the weighting function.

$$
\mathrm{pv}=\sum_{i, j} p v_{i j}^{+} \pi^{+}\left(\omega_{j}\right)+\sum_{i, j} \mathrm{pv}_{i j}^{-} \pi^{-}\left(\omega_{j}\right)
$$

3.2.2. Decision Matrix. To develop the CADM model, a decision matrix should be established to describe the number of $n$ available collision avoidance schemes $\left\{U_{1}, U_{2}, \ldots, U_{n}\right\}$. For instance, under an encounter situation shown in Figure 6, there are three available collision avoidance schemes $\left\{U_{1}, U_{2}, U_{3}\right\}$. The first scheme $U_{1}=\left(t_{1}, P_{1}, \theta_{1}, d_{\text {accl }}\right)$ means when the OS is moving to the position $P_{1}$ at time $t_{1}$, the collision risk is unacceptable for a MASS with the RA of caution. The MASS makes a course change $\theta_{1}$ and finally passes the TS with an accepted distance of $d_{\mathrm{accl}}$ (green line in Figure 6). Similarly, the avoidance schemes for neutral RA and aggressive RA are $U_{2}=\left(t_{2}, P_{2}, \theta_{2}, d_{2}\right)$ and $U_{3}=\left(t_{3}, P_{3}, \theta_{3}, d_{3}\right)$, respectively, leading to accepted distance of $d_{\text {acc2 }}$ (yellow line in Figure 6) and $d_{\mathrm{acc} 3}$ (red line in Figure 6).

Each $U$ contains the number of $m$ decision-making indicators $\left\{x_{1}, x_{2}, \ldots, x_{m}\right\}$. The decision-making matrix $X$ can be represented as follows:

$$
X=\left[\begin{array}{cccc}
x_{11} & x_{12} & \cdots & x_{1 m} \\
x_{21} & x_{22} & \cdots & x_{2 m} \\
\vdots & \vdots & \ddots & \vdots \\
x_{n 1} & x_{n 2} & \cdots & x_{n m}
\end{array}\right] .
$$

In the decision matrix, each indicator $\left(x_{1}=\mathrm{SV}\right.$, $x_{2}=$ Dev, and $x_{3}=$ Sta) that is obtained from RA assessor needs to be normalized into an interval of $[-1,1]$ to acquire a normalized decision matrix $\mathbf{R}$ by using linear transformation equations, in which

$$
Z_{j}=\frac{1}{n} \sum_{i=1}^{n} x_{i j}
$$

For indicator of Dev,

$$
r_{i j}=\frac{z_{j}-x_{i_{j}}}{\max \left\{\max _{j}\left(x_{i j}\right)-z_{j}, z_{j}-\min _{j}\left(x_{i j}\right)\right\}} .
$$

For indicator of Sv and Sta, 


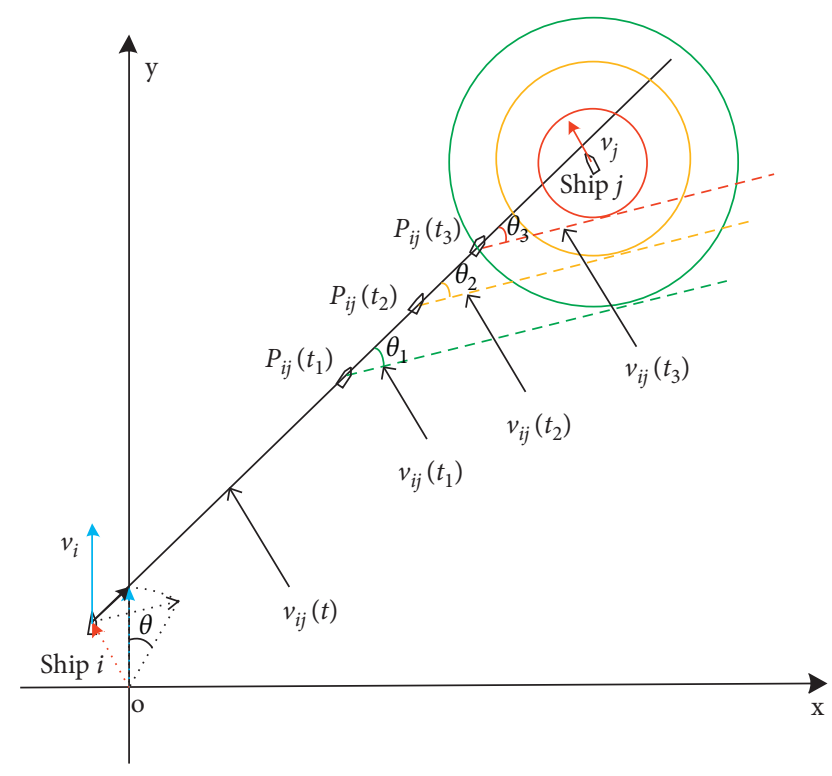

FIgURE 6: An example of ship encounter situation.

$$
r_{i j}=\frac{x_{i_{j}}-z_{j}}{\max \left\{\max _{j}\left(x_{i j}\right)-z_{j}, z_{j}-\min _{j}\left(x_{i j}\right)\right\}} .
$$

3.2.3. Reference Points. It can be noted that the used reference points are crucial for evaluating the performance of the indicators in a PT-based model. For a CADM model, the selections of the reference points are associated with OPS. Once the reference points are selected, the optional decision scheme can be evaluated by yielding prospect values between the positive (i.e., gains) and negative (i.e., losses). In other words, the selection of reference points in the CADM model means the expression of OOWs' knowledge and experience.

As each OOW has his/her own experience to determine the gains and the losses under different encounter situations, setting a global value for universal scenarios is not reasonable. Thus, the CADM model applies a grey correlation analysis technology to determining the reference point under different situations. The technology is based on the method of proximity measure of similarity, which can calculate the degree of association between factors and use the parameter to indicate the reference point. The correlation coefficient for each indicator under different collision avoidance schemes can be calculated as follows:

$$
\begin{aligned}
& \xi_{i j}^{+}=\frac{\min _{i} \min _{j}\left|r_{i j}-r_{j}^{+}\right|+\rho \max _{i} \max _{j}\left|r_{i j}-r_{j}^{+}\right|}{\left|r_{i j}-r_{j}^{+}\right|+\rho \max _{i} \max _{j}\left|r_{i j}-r_{j}^{+}\right|}, \\
& \xi_{i j}^{-}=\frac{\min _{i} \min _{j}\left|r_{i j}-r_{j}^{-}\right|+\rho \max _{i} \max _{j}\left|r_{i j}-r_{j}^{-}\right|}{\left|r_{i j}-r_{j}^{-}\right|+\rho \max _{i} \max _{j}\left|r_{i j}-r_{j}^{-}\right|},
\end{aligned}
$$

where $\quad r_{j}^{+}=\max \left\{r_{i j} \mid 1 \leq i \leq n\right\}, r_{j}^{-}=\min \left\{r_{i j} \mid 1 \leq i \leq n\right\}, j=$ $1,2, \ldots, m$, and $\rho \in[0,1]$, while positive and negative reference points for each indicator are identified as follows:

$$
\begin{array}{ll}
\xi_{j}^{+}=\max \left\{\xi_{i j}^{+} \mid 1 \leq i \leq n\right\}, & j=1,2, \ldots, m, \\
\xi_{j}^{-}=\max \left\{\xi_{i j}^{-} \mid 1 \leq i \leq n\right\}, & j=1,2, \ldots, m .
\end{array}
$$

3.2.4. Composite Prospect Value. Based on PT, the composite prospect value of each scheme can be calculated by using the following equation:

$$
\mathrm{pv}=\sum_{i, j} \mathrm{pv}_{i j}^{+} \pi^{+}\left(\omega_{j}\right)+\sum_{i, j} \mathrm{pv}_{i j}^{-} \pi^{-}\left(\omega_{j}\right)
$$

and the aggregated prospect value can be calculated by using the objective function:

$$
\max \mathrm{pv}=\sum_{i=1}^{m} \sum_{j=1}^{n} \mathrm{pv}_{i j}^{+} \pi^{+}\left(\omega_{j}\right)+\sum_{i=1}^{m} \sum_{j=1}^{n} \mathrm{pv}_{i j}^{-} \pi^{-}\left(\omega_{j}\right),
$$

where the weight $\omega^{*}=\left(\omega_{1}^{*}, \omega_{2}^{*}, \ldots, \omega_{m}^{*}\right)$ for each indicator is obtained based on the user's RA and OPS, in which s.t. $\left\{\begin{array}{l}0 \leq \omega_{j} \leq 1 \\ \sum_{j} \omega_{j}=1 \text {. It can be assigned by expert judgements and }\end{array}\right.$ linear programming.

\section{Case Study}

To validate the proposed system, the simulations are carried out to test the effectiveness of the RA-CADM model. The simulation test contains two typical ship encounter scenarios, which are carried out in MATLAB software using the i7-8550U CPU and 12GB RAM. In the simulations, one of the scenarios describes a MASS encounters an individual manned vessel and another presents a multiple encounter situation.

4.1. Ship Manoeuvrability Simulation. The Nomoto model is used to simulate the ship movement when the ships take a collisions avoidance action. To simplify the model, the impacts from wind and current are not taken into consideration. Consequently, the ship course changes are simulated by using the following equation:

$$
T \times \dot{r}+r=K \times \delta
$$

where $T$ is the constant time for a ship changing its course and $K$ is the rudder gain, $r$ is the angular speed, and $\delta$ is the rudder angle. The course change after time of $t$ can be calculated as

$$
\theta=K \times \delta_{0} \times\left(t-T+T \times e^{-(t / T)}\right),
$$

while the $K$ can be calculated with $K=V / L$ and $T=2 \times L / V$ based on experience. 
4.2. Scenario I: Pairwise Vessel Encounter. Based on the simulations, the first target is to test the typical individual collision avoidance scenario. As shown in Table 1, the initial encounter scenario is that the OS (i.e., MASS) is heading north along the planned route, while the TS comes from the starboard side with bearing of $049^{\circ}$. According to COLREGS, the MASS should perform its duty of giving way to the coming ship. Then, the simulation assumes that both ships are equipped with AIS, which is able to broadcast ship dynamic information with a period of 10 seconds when a ship remains its course or with a period of 3 seconds when the ship is taking a course change.

The initial information for both ships is given in Table 1. The speeds for OS and TS are 11 and 13 knots, respectively, the initial course for the MASS is $000^{\circ}$, and the initial course for the TS is $275^{\circ}$. The relative distance between the two ships is 7 nautical miles and the lengths for them are 225 meters and 189 meters. In addition, two vessels are encountered at the open and calm sea.

To avoid collision, the MASS tries to keep clear of all the TS under six possible accepted distances, where $d_{\mathrm{acc}} \in(1.0,1.2$, $1.4,1.6,1.8,2.0)$. In terms of the course changes, six degrees are provided for the MASS from low to high: $\theta \in\left(20^{\circ}, 25^{\circ}, 30^{\circ}, 35^{\circ}, 40^{\circ}, 45^{\circ}\right)$, which reflect the RA of OOWs. In order to test the collision avoidance behavior more apparently, Table 2 offers the details on the collision avoidance schemes, including the accepted distance, course of changes, action times, recover time, and initial route deviation.

As the examples, the OS/ trajectory for scheme of $U_{1}^{1}$ (the blue trajectory) and $U_{6}^{1}$ (the green trajectory) and TS's trajectory (pink) are demonstrated in Figure 7. In the scheme of $U_{1}^{1}$, the MASS decides to pass the TS with an accepted distance of $1.0 \mathrm{~nm}$, thus it needs to change the course to $020^{\circ}$ at the action time of 12.1 minutes. After it passed the TS, the MASS recovers its course to $000^{\circ}$ at 26.4 minutes. The whole collision avoidance action takes 14.3 minutes. In the scheme of $U_{6}^{1}$, the MASS changes its course to $045^{\circ}$ at 8.8 minutes to pass the TS with an acceptable distance of $2.0 \mathrm{~nm}$. After passing the TS, the MASS recovers its course to $000^{\circ}$ at 24.3 minutes. The total action time for scheme $\mathrm{U}_{6}$ is 15.5 minutes.

The RA-CADM model is used to evaluate the collision avoidance schemes that are given in Table 3 and the most suitable scheme is selected for the MASS under different risk preferences (i.e., caution, neutral, and aggressive). The overall process includes five steps, taking aggressive as an example (Tversky, 1992) $(\eta=2.25, \alpha=\beta=0.88, \gamma=0.61$, $\tau=0.69)$ :

Step 1: calculating the RA indicator for each scheme. The values of RA indicator for the scheme can be calculated using equations (1)-(5). Using the scheme $U_{1}$ as an example, the RA values of safety, efficiency, and stationarity can be calculated as $\mathrm{SV}_{U 1}=0.8 \times$ $(1.0 / 2.0)^{0.65}+0.2 \times(13.5 / 25)=0.618, \quad \operatorname{Dev}_{U 1}=11 \times$ $26.4-12.1 / 60 \times \sin 20^{\circ}=0.897$, and $\operatorname{Sta}_{U 1}=\pi-20^{\circ}$ $\times \pi / 180^{\circ}=2.793$, where $d_{1}=1.0, t_{1}=25, \mathrm{TCPA}_{U 1}=$ 25.6, and $\mathrm{TCPA}_{U 1}=25.6-12.1=13.5$. Similarly, the values of the RA indicators for the other 5 schemes are calculated to construct the decision-making matrix $\mathbf{X}$, which is
TABLE 1: Scenario I: encounter information.

\begin{tabular}{lcc}
\hline & OS & TS \\
\hline SOG (knots) & 11 & 13 \\
COG & $000^{\circ}$ & $275^{\circ}$ \\
Distance (nm) & 7 & 7 \\
Relative bearing $\left(^{\circ}\right)$ & - & 049 \\
Ship length (meters) & 225 & 189 \\
\hline
\end{tabular}

$$
\mathbf{X}=\left[\begin{array}{lll}
0.618 & 0.897 & 2.793 \\
0.692 & 1.178 & 2.705 \\
0.759 & 1.448 & 2.618 \\
0.822 & 1.693 & 2.531 \\
0880 & 1.862 & 2.443 \\
0.934 & 2.009 & 2.356
\end{array}\right]
$$

Step 2: normalization. The RA indicator values in the $\mathbf{X}$ are then normalized to acquire the normalized decision matrix $\mathbf{R}$, in which equations (11)-(13) are used to calculate the normalized RA values for safety, efficiency, and stationarity, respectively. As a result,

$$
R=\left[\begin{array}{lll}
r_{11} & r_{12} & r_{13} \\
r_{21} & r_{22} & r_{23} \\
r_{31} & r_{32} & r_{33} \\
r_{41} & r_{42} & r_{43} \\
r_{51} & r_{52} & r_{53} \\
r_{61} & r_{62} & r_{63}
\end{array}\right]=\left[\begin{array}{ccc}
-1.000 & 1.000 & 1.000 \\
-0.552 & 0.545 & 0.600 \\
-0.150 & 0.107 & 0.200 \\
0.225 & -0.289 & -0.200 \\
0.575 & -0.562 & -0.600 \\
0.903 & -0.801 & -1.000
\end{array}\right] .
$$

Step 3: identifying the reference points. To evaluate the performance of the RA indicators in the RA-CADM model, the maximum and minimum values of each indicator are selected from the normalized decision matrix $\mathbf{R}$. The positive set (i.e., the maximum value set) is $r^{+}=\{0.903,1,1\}$ and negative set (i.e., the minimum value set) is $r^{-}=\{-1,-0.801,-1\}$. Using equations (14) and (15), the $r_{11}$ in the $R$ is divided to two correlation coefficient values $\xi_{11}^{+}$and $\xi_{11}^{-}$, where

$$
\begin{aligned}
\xi_{11}^{+} & =\frac{\min _{i} \min _{j}\left|r_{i j}-r_{j}^{+}\right|+\rho \max _{i} \max _{j}\left|r_{i j}-r_{j}^{+}\right|}{\left|r_{i j}-r_{j}^{+}\right|+\rho \max _{i} \max _{j}\left|r_{i j}-r_{j}^{+}\right|} \\
& =\frac{0+0.5 \times 2}{|-1-0.903|+0.5 \times 2}=0.345, \\
\xi_{11}^{-} & =\frac{\min _{i} \min _{j}\left|r_{i j}-r_{j}^{-}\right|+\rho \max _{i} \max _{j}\left|r_{i j}-r_{j}^{-}\right|}{\left|r_{i j}-r_{j}^{-}\right|+\rho \max _{i} \max _{j}\left|r_{i j}-r_{j}^{-}\right|} \\
& =\frac{0+0.5 \times 2}{|-1+1|+0.5 \times 2}=1 .
\end{aligned}
$$


TABLE 2: Scenario I: collision avoidance schemes.

\begin{tabular}{lccccc}
\hline No. of schemes & Accepted distance $(\mathrm{nm})$ & $\begin{array}{c}\text { Change of } \\
\text { course (degrees) }\end{array}$ & Action time (minutes) & Recover time (minutes) & Initial route deviation (nm) \\
\hline$U_{1}^{1}$ & 1.0 & 20 & 12.1 & 26.4 & 0.9 \\
$U_{2}^{1}$ & 1.2 & 25 & 10.8 & 26.0 & 1.2 \\
$U_{3}^{1}$ & 1.4 & 30 & 10.0 & 25.8 & 1.5 \\
$U_{4}^{1}$ & 1.6 & 35 & 9.4 & 25.5 & 1.7 \\
$U_{5}^{1}$ & 1.8 & 40 & 9.0 & 24.8 & 1.8 \\
$U_{6}^{1}$ & 2.0 & 45 & 8.8 & 24.3 & 2.0 \\
\hline
\end{tabular}

TABLE 3: Scenario II: initial encounter information.

\begin{tabular}{lccc}
\hline & OS & TS1 & TS2 \\
\hline SOG (knots) & 14 & 10 & 10 \\
COG & $000^{\circ}$ & $210^{\circ}$ & $225^{\circ}$ \\
Distance $(\mathrm{nm})$ & - & 6 & 8 \\
Relative bearing $\left(^{\circ}\right)$ & - & 027 & 021 \\
DCPA (nm) & - & 1.42 & 0.50 \\
\hline
\end{tabular}
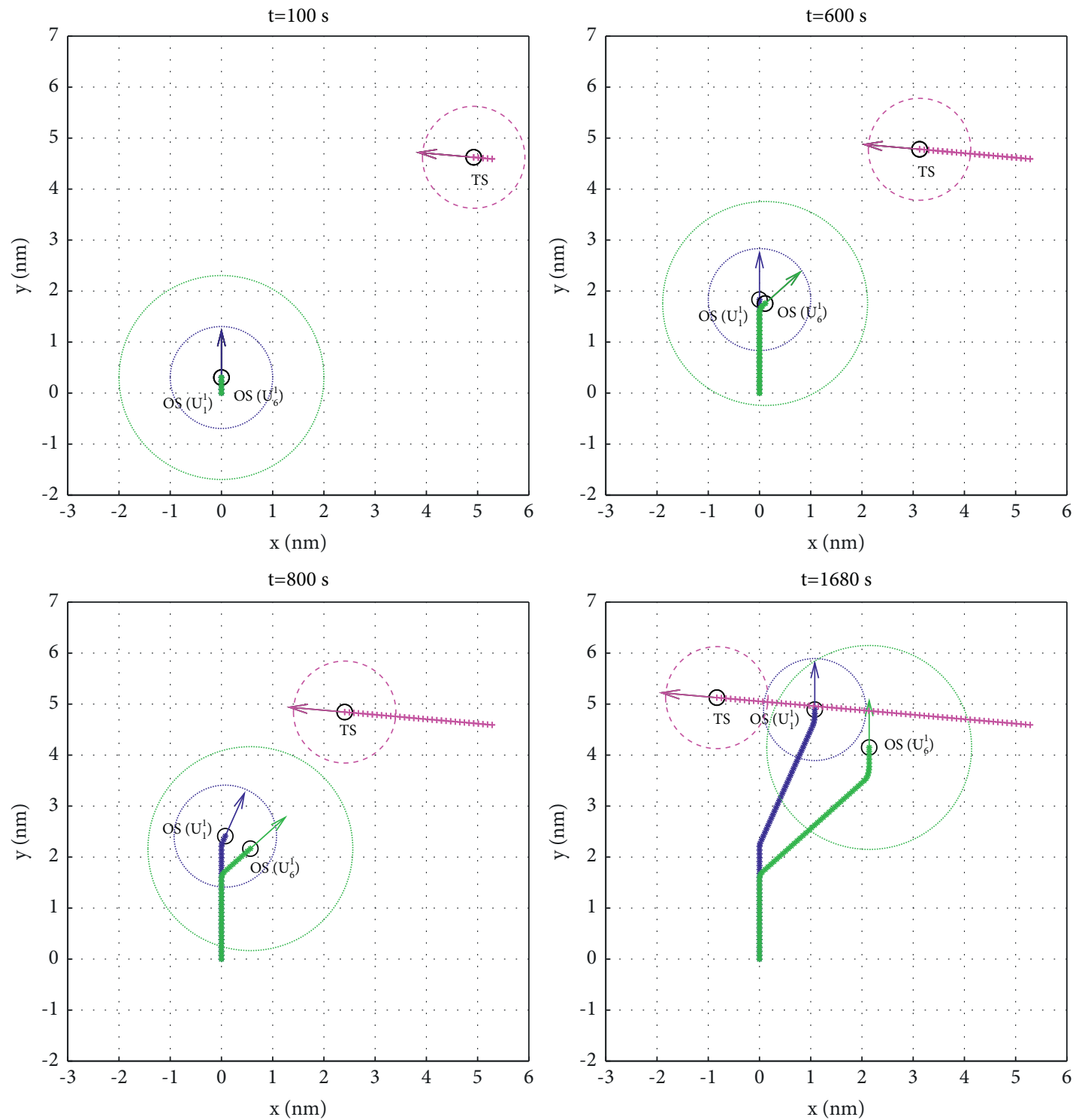

Figure 7: Ship trajectories in Case 1. 
Similarly, the normalized decision matrix $\mathbf{R}$ is converted to the positive correlation coefficient matrix,

$$
\xi_{i j}^{+}=\left[\begin{array}{lll}
0.345 & 1.000 & 1.000 \\
0.407 & 0.687 & 0.714 \\
0.487 & 0.528 & 0.556 \\
0.596 & 0.437 & 0.455 \\
0.753 & 0.390 & 0.385 \\
0.800 & 0.357 & 0.333
\end{array}\right],
$$

and the negative correlation coefficient matrix,

$$
\xi_{i j}^{-}=\left[\begin{array}{lll}
1.000 & 0.357 & 0.333 \\
0.691 & 0.426 & 0.385 \\
0.541 & 0.524 & 0.455 \\
0.450 & 0.661 & 0.556 \\
0.388 & 0.807 & 0.714 \\
0.345 & 1.000 & 1.000
\end{array}\right] .
$$

In $\xi_{i j}^{+}$and $\xi_{i j}^{-}$, the maximum and the minimum values in each column are selected as the reference points to calculate the positive prospect value $\mathrm{pv}^{+}$and negative prospect value $\mathrm{pv}^{-}$by using equations (6) and (7). In the calculation, the risk preference parameters are defined as an aggressive RA that is $\alpha=\beta=0.88$. Thereby, $\quad\left\{\begin{array}{l}\mathrm{pv}_{11}^{+}=(1-1)^{0.88}=0 \\ \mathrm{pv}_{12}^{+}=(1-0.357)^{0.88}=0.678 \\ \mathrm{pv}_{13}^{+}=(1-0.333)^{0.88}=0.7\end{array} \quad\right.$ and $\left\{\begin{array}{l}\mathrm{pv}_{11}^{-}=-2.25 \times(1-0.345)^{0.88}=-1.552 \\ \mathrm{pv}_{12}^{-}=-2.25 \times(1-1)^{0.88}=0 \\ \mathrm{pv}_{13}^{-}=-2.25 \times(1-1)^{0.88}=0\end{array}\right.$. As results, the positive prospect matrix $\mathrm{pv}^{+}=\left[\begin{array}{ccc}0 & 0.678 & 0.700 \\ 0.356 & 0.613 & 0.652 \\ 0.504 & 0.520 & 0.587 \\ 0.591 & 0.386 & 0.490 \\ 0.649 & 0.235 & 0.332 \\ 0.690 & 0 & 0\end{array}\right]$. and the negative prospect matrix $\quad \mathrm{pv}^{-}=\left[\begin{array}{ccc}-1.552 & 0 & 0 \\ -1.420 & -0.809 & -0.747 \\ -1.250 & -1.161 & -1.102 \\ -1.014 & -1.357 & -1.320 \\ -0.657 & -1.456 & -1.468 \\ -0.546 & -1.525 & -1.575\end{array}\right] \quad$ are obtained.

Step 4: assigning weights to the indicators. With aids of a linear interactive optimize software program (LINGO 15.0), the optimal weight assignments $\omega^{*}=$ $\left(\omega_{1}, \omega_{2}, \omega_{3}\right)$ for each RA indicator can be simulated. To set the maximum target function as MAX $=(0+$ $0.356+0.504+0.591+0.649+0.69) \times\left(w_{1}^{0.61} / w_{1}^{0.61}+\right.$ $\left.\left(1-w_{1}\right)^{0.61}\right)^{1 / 0.61}+(-1.552-1.42-1.25-1.014-$ $0.657-0.546) \times\left(w_{1}^{0.69} / w_{1}^{0.69}+\left(1-w_{1}\right)^{0.69}=\right)^{1 / 0.69}$. while defining the limitation of outputs as, s.t. $\left\{\begin{array}{l}0 \leq \omega_{j} \leq 1 \\ \sum_{j} \omega_{j}=1 \\ w_{1} \geq 0.5\end{array}\right.$, the weight assignments are generated as $\omega^{*}=\{0.5,0.4,0.1\}$.

Step 5: calculating the composite prospect value for each collision avoidance scheme. The composite prospect values for each scheme are calculated by aggregating the above information using equation (18). Thereby, $\mathrm{pv}_{U 1}=$ $-0.25, \mathrm{pv}_{U 2}=-0.53, \mathrm{pv}_{U 3}=-0.65, \mathrm{pv}_{U 4}=-0.70, \mathrm{pv}_{U 5}$ $=-0.69$, and $\mathrm{pv}_{U 6}=-0.86$. Based on the results, the optimized ranking for an aggressive RA user,

$$
\text { Aggressive } \sim\left(U_{1}^{1}>U_{2}^{1}>U_{3}^{1}>U_{5}^{1}>U_{4}^{1}>U_{6}^{1}\right) .
$$

Similarly, if the RA for the MASS is set as neutral, the parameter set of $\eta=2.25, \alpha=\beta=0.99$, and $\gamma=\tau=0.99$ should be used according to previous studies [55]. The optimum collision avoidance schemes are resequenced as

$$
\text { Neutral } \sim\left(U_{4}^{1}>U_{5}^{1}>U_{3}^{1}>U_{2}^{1}>U_{1}^{1}>U_{6}^{1}\right)
$$

where the composite prospect values for the schemes are $\mathrm{pv}_{U 1}=-0.67, \mathrm{pv}_{U 2}=-0.66, \mathrm{pv}_{U 3}=-0.62, \mathrm{pv}_{U 4}=-0.50$, $\mathrm{pv}_{U 5}=-0.55$, and $\mathrm{pv}_{U 6}=-0.73$.

Meantime, for RA is cautious, the parameter value assignment is $\eta=3.5, \alpha=1.21, \beta=1.02, \gamma=0.55$, and $\tau=$ 0.49 [56]. The composite prospect values are calculated as $p v_{U 1}=-1.25, p v_{U 2}=-1.22, p v_{U 3}=-1.20, \quad p v_{U 4}=-1.10$, $p v_{U 5}=-0.89$, and $p v_{U 6}=-0.87$. The collision avoidance schemes are ranked as

$$
\text { Cautious } \sim\left(U_{6}^{1}>U_{5}^{1}>U_{4}^{1}>U_{3}^{1}>U_{2}^{1}>U_{1}^{1}\right) .
$$

4.3. Scenario II: Multiple-Vessel Encounter. As the proposed RA-CADM model is able to deal with multiple-vessel encounter situations, the second scenario simulates an encounter situation that involves 2 TSs (as shown in Figure 8) and the ship details are given in Table 3.

As shown in Figure 8, two TSs come from starboard of the MASS, in which the TS1 will keep clear of the MASS and the TS2 will cross the OS' safety distance and lead to high risk of collision. To avoid the urgent risk of collision, the MASS needs to select an optimal collision scheme that not only keeps clear of the TSs but also ensures the efficiency of the action. As stated in the COLREGS that a give-way ship shall change course to starboard to avoid collision, thereby only two collision avoidance strategies can be considered by the MASS: (1) changing course to starboard with large degree to keep clear the TSs but suffering bigger deviation distance (i.e., low efficiency) or (2) remaining its course before passing the TS1 and then turning starboard to avoid the TS2 so that the deviation distance will be relatively small. On such a basis, the available collision avoidance schemes are shown in Table 4, in which the former three schemes $U_{1}^{2}$, 


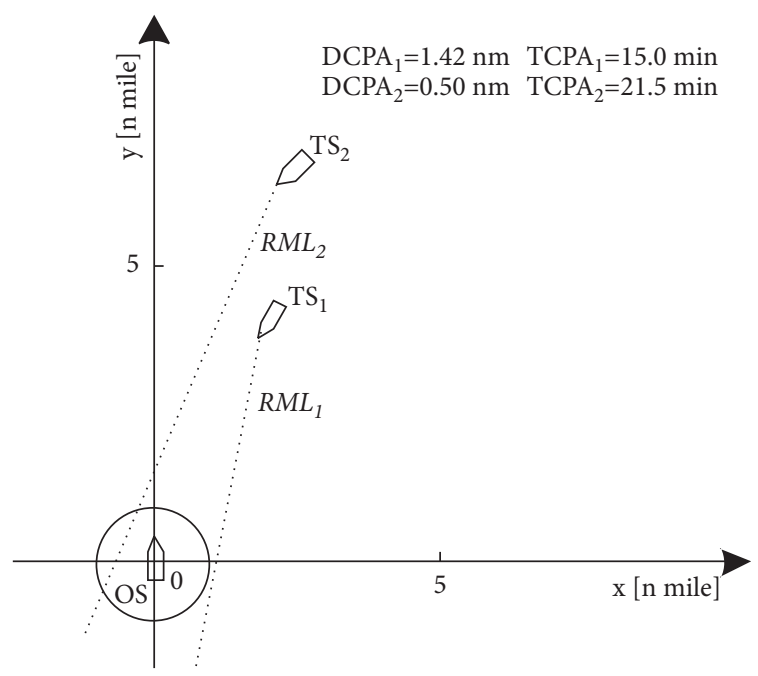

Figure 8: The multiple-vessel encounter situation.

TABLE 4: Scenario II: collision avoidance scheme.

\begin{tabular}{lcccc}
\hline Scheme & Accepted distance $(n$ mile $)$ & Change of course (degree) & Action time (minutes) & Recover time (minutes) \\
\hline$U_{1}^{2}$ & 1.0 & 20 & 13.6 & 23.6 \\
$U_{2}^{2}$ & 1.2 & 30 & 13.8 & 23.7 \\
$U_{3}^{2}$ & 1.4 & 40 & 0.3 & 23.6 \\
$U_{4}^{2}$ & 1.6 & 50 & 0.3 & 25.0 \\
$U_{5}^{2}$ & 1.8 & 55 & 0.2 & 24.8 \\
$U_{6}^{2}$ & 2.0 & 60 & & 24.8 \\
\hline
\end{tabular}

$U_{2}^{2}$, and $U_{3}^{2}$ are the smaller deviation strategy and the subsequent three $U_{4}^{2}, U_{5}^{2}$, and $U_{6}^{2}$ are the large deviation strategy.

The simulation results of the TS1 (pink), TS2 (black), and MASS's trajectories by applying the schemes $U_{1}^{2}, U_{1}^{2}$ (blue) and $U_{6}^{2}, U_{6}^{2}, U_{6}^{2}$ (green) are given in Figure 9.

By using the same step introduced above, the composite prospect value for each collision avoidance scheme in the second scenario can be obtained that $\mathrm{pv}_{U 1}=-0.25, \mathrm{pv}_{U 2}=$ $-0.44, \mathrm{pv}_{U 3}=-0.53, \mathrm{pv}_{U 4}=-0.71, \mathrm{pv}_{U 5}=-0.70$, and $\mathrm{pv}_{U 6}=-0.87$. Therefore, the collision avoidance schemes for aggressive RA are ranked as

$$
\text { Aggressive } \sim\left(U_{1}^{2}>U_{2}^{2}>U_{3}^{2}>U_{5}^{2}>U_{4}^{2}>U_{6}^{2}\right) .
$$

Similarly, the collision avoidance schemes of neutral and cautious officers are ranked as

$$
\begin{gathered}
\text { Neutral } \sim\left(U_{3}^{2}>U_{2}^{2}>U_{4}^{2}>U_{1}^{2}>U_{5}^{2}>U_{6}^{2}\right), \\
\text { Cautious } \sim\left(U_{6}^{2}>U_{5}^{2}>U_{4}^{2}>U_{3}^{2}>U_{2}^{2}>U_{1}^{2}\right) .
\end{gathered}
$$

\section{Results and Discussion}

5.1. Indicator Values under Different RA. The case study results show that the RA-CADMS is capable of aiding the MASS to select the optimal collision avoidance schemes based on the RA and risk tolerance. To validate the rationality of the outputs from the model, this section discusses the variation of the RA indicators in terms of a dynamic prospective.
5.1.1. $R A=$ Aggressive. As shown in Figure 10, when setting the RA for a MASS to avoid collision as aggressive, the schemes of $U_{1}^{1}$ and $U_{1}^{2}$ are chosen as the optimal action for the MASS under scenarios I and II, respectively. In the scenario I, the dynamic values of three RA indicators are reported in Figure 10(a). It can be noted the MASS takes course change at time $725 \mathrm{~s}$ when the SV is relatively low (0.4). After the action is made, the SV for the MASS is increasing to 0.6 , but the Dev began to grow and reached $1.0 \mathrm{~nm}$ in the end. In scenario II (see Figure 10(b)), the MASS takes actions at time $845 \mathrm{~s}$ and the SV is increased from 1.05 to 1.20 , causing the deviation of $0.9 \mathrm{~nm}$.

5.1.2. $R A=$ Neutral. When $\mathrm{RA}$ is set as neutral, the RACADMS selects the schemes $U_{4}^{1}$ and $U_{3}^{2}$ as two optimal decisions for the MASS under scenario I and scenario II. As shown in Figure 11(a), the MASS chooses to take collision avoidance action at time $560 \mathrm{~s}$ when SV is 0.43 . As a result, the SV significantly raised to 0.79 and the Dev increased to 1.7. In scenario II (see Figure 11(b), the MASS takes action at time $835 \mathrm{~s}$ and the $\mathrm{SV}$ is increased from 1.05 to 1.25 , leading to a Dev of $1.7 \mathrm{~nm}$.

5.1.3. $R A=$ Cautious. Similarly, when $\mathrm{RA}=$ cautious, the optimal schemes for the MASS are $U_{6}^{1}$ (for scenario I) and $U_{4}^{2}$ (for scenario II). As shown in Figure 12(a), in scenario I, the action time for the MASS is $520 \mathrm{~s}$ when SV is 0.45 ; after the collision avoidance action is took, the SV increases to maximum, but the Dev is also relatively large $(\mathrm{Dev}=2.0)$. In 

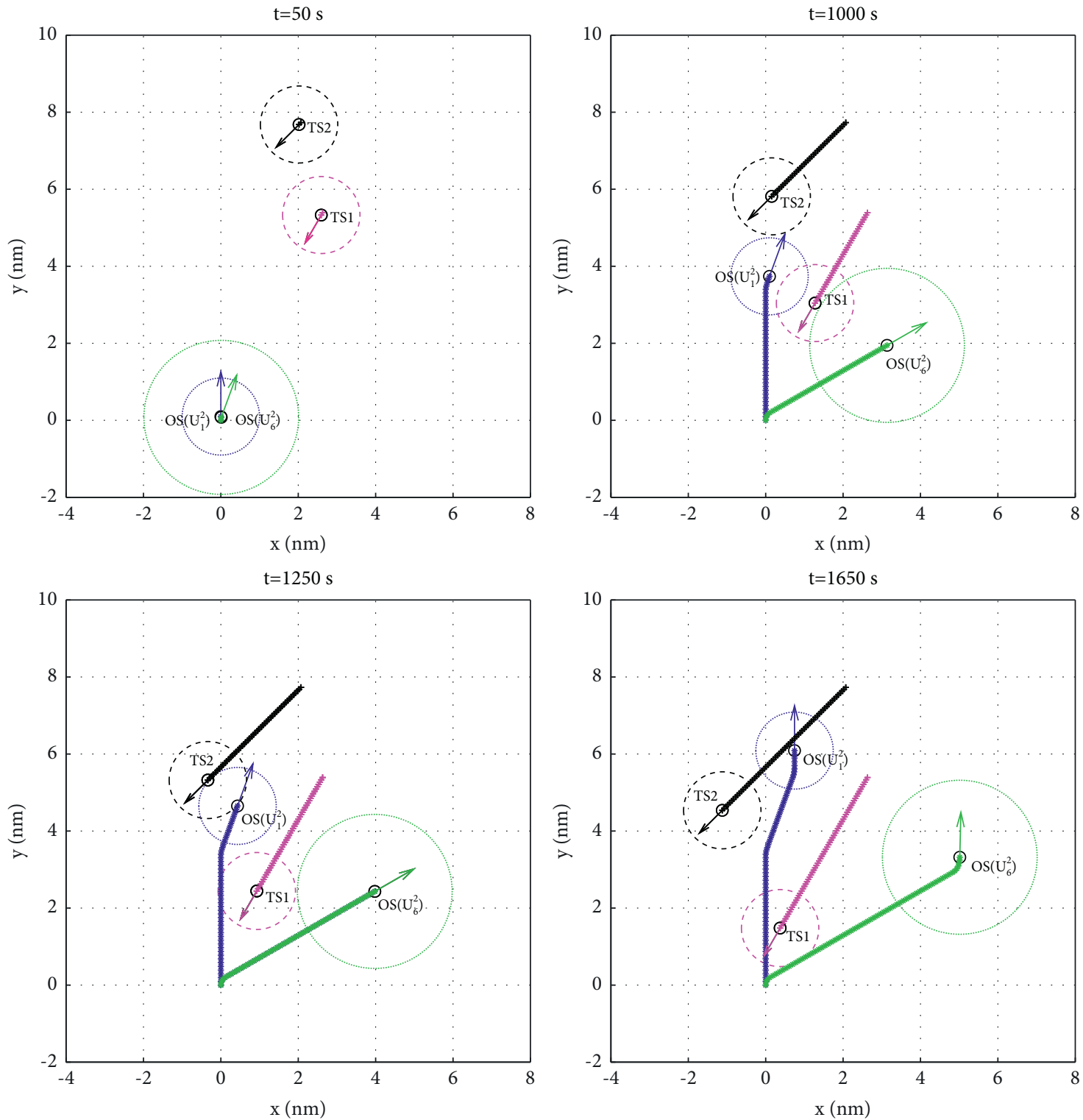

FIGURE 9: Ship trajectories in Case 2.

scenario II, Figure 12(b), the MASS chooses to avoid two TSs at the initial time of the simulation. For that moment, the SV is 1.6. The action causes a large route deviation, which is the largest among all the schemes $(\operatorname{Dev}=5)$.

5.2. Characteristics of Different $R A$. The simulation results demonstrate that different RA setting in the MASS leads to the different collision avoidance actions, proving a close relationship among them. To characterize the avoidance actions under different RA can provide better understanding for MASS designer/controllers.

Table 5 reports the details of the scheme selections. Five factors are considered, including the action time, SV values before action, SV values after action, and SV increment and deviation. Based on Table 4, the following findings are concluded:
(1) For MASS, the more conservative it is, the easier it is to take collision avoidance action earlier. It is evident that the cautious RA selects the earliest action time (520 seconds) in scenario I, which is 205 seconds earlier than the scheme that is selected by the aggressive RA. This phenomenon is more apparent in scenario II as the scheme $U_{6}^{2}$ takes action at initial time, while the $U_{1}^{2}$ takes action at 845 seconds. This is due to the risk tolerance under different RA. For instance, in scenario I, the minimum accepted SV for the aggressive RA is 0.4 , while the minimum accepted SV for cautious is 0.45 . The high tolerance of risk causes later actions.

(2) Followed by the first findings, this study notes that the safety levels under different RA are different. For instance, in the scheme $U_{1}^{1}$ of scenario I, the SV raises to maximum (i.e., $\mathrm{SV}=1$ ) after the collision 


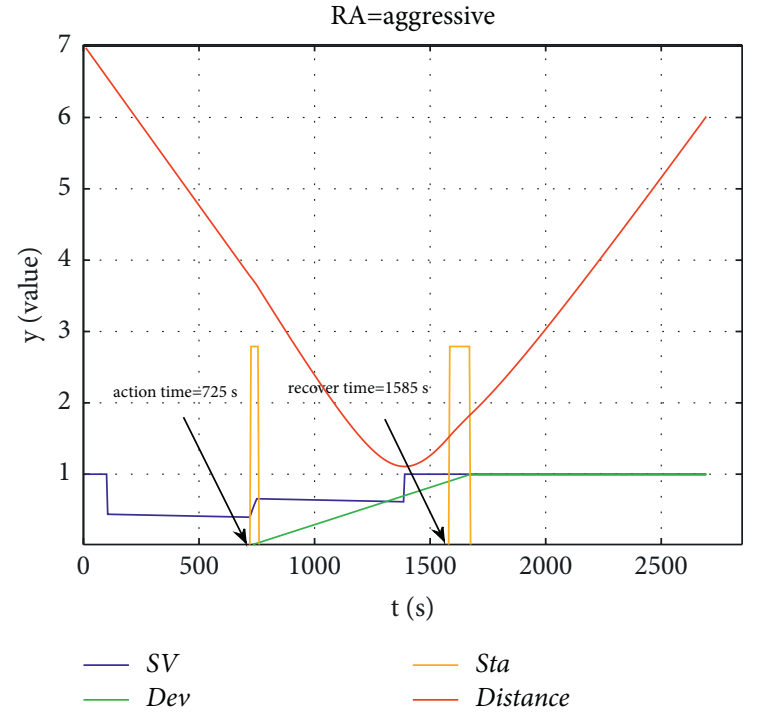

(a)

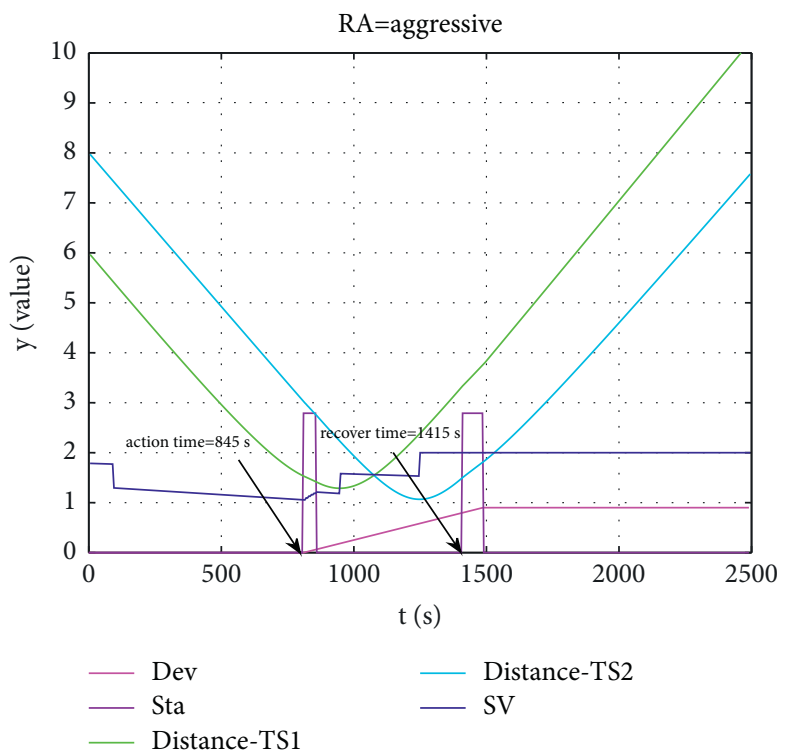

(b)

FIGURE 10: Results of decision indicators with aggressive officers in (a) scenario I and (b) scenario II.

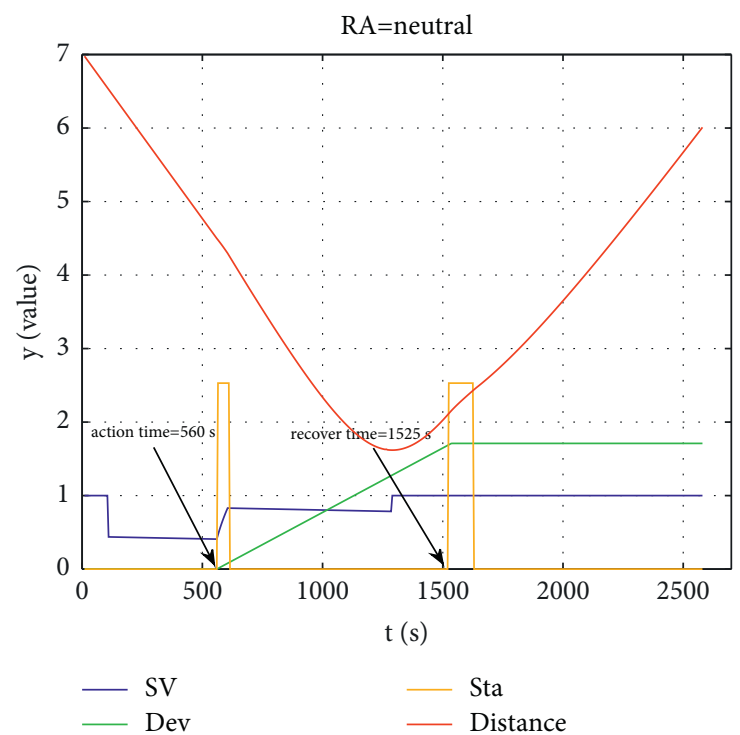

(a)

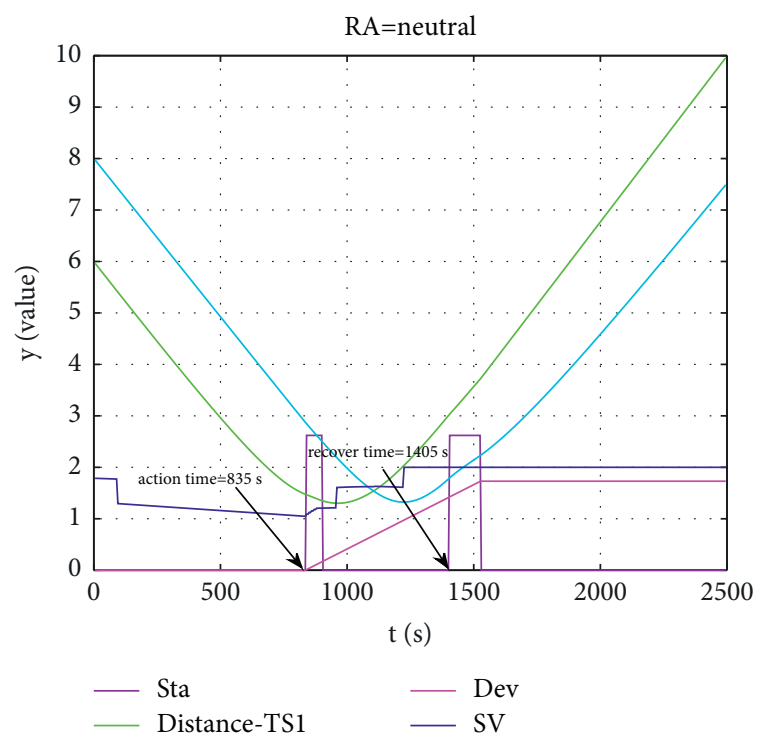

(b)

FIGURE 11: Results of decision indicators with neutral officers in (a) scenario I and (b) scenario II.

avoidance action is taken by a cautious RA, with a significant increasing on SV (from 0.45 to 0.55 ). In contrast, the MASS with high-risk tolerance (i.e., aggressive) accepts SV $=0.6$ as the safety level. It means the action taken by aggregative RA is riskier.

(3) Under different risk appetites, the composite prospect value of the same collision avoidance schemes for the same encountering scenario is different. The composite prospect value of the aggressive crew is larger than that of the cautious crew, for the aggressive pursuing greater prospect value, but the prospect value of each scheme is negative in any type of officers, indicating that there exists expected deviation compared to ideal decision.

(4) In practice, MASSs can recommend the officers optimal schemes according to the specific risk appetite types of different operators, which can not only ensure the safety and economy of ship collision avoidance action but also be well understood and accepted by the OOWs. 


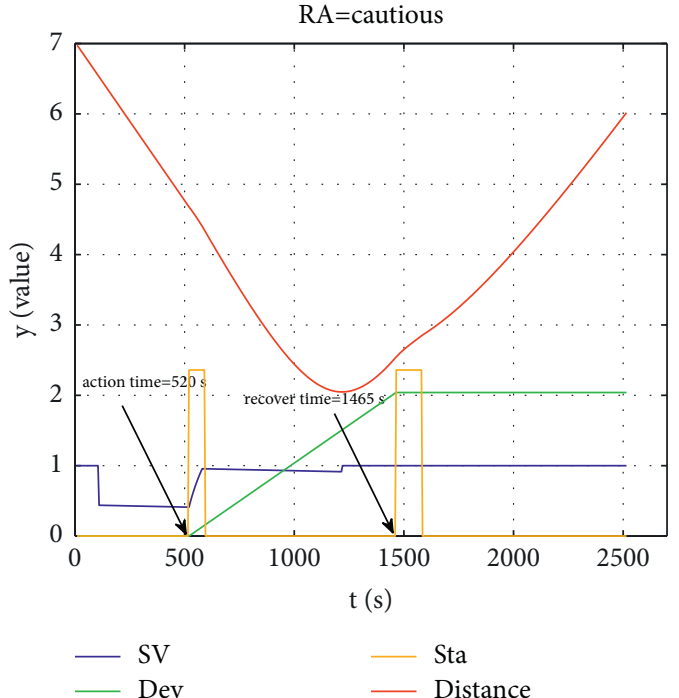

(a)

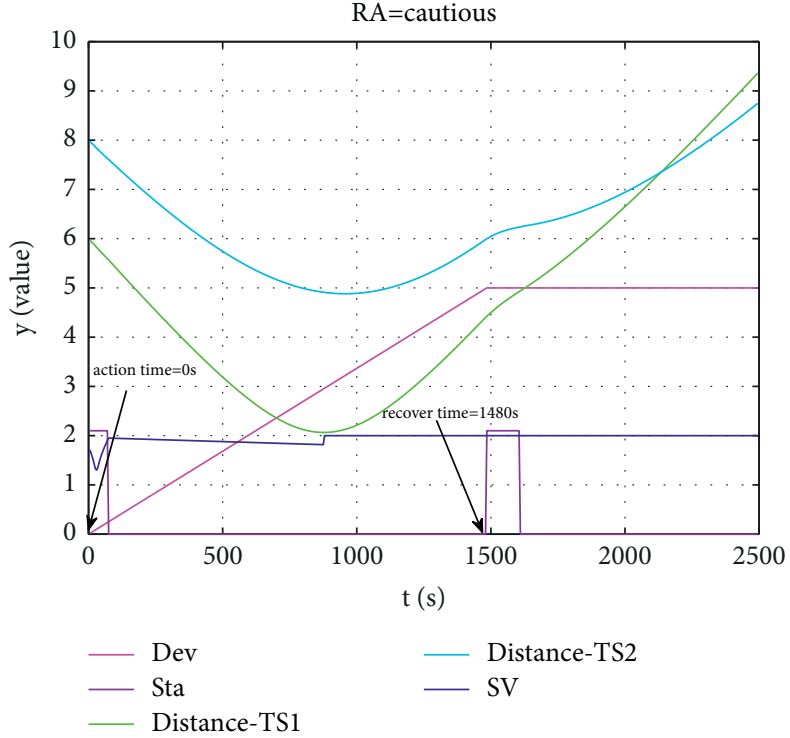

(b)

FIGURE 12: Results of decision indicators with cautious officers in (a) scenario I and (b) scenario II.

TABle 5: Results under different RA.

\begin{tabular}{|c|c|c|c|c|c|c|c|}
\hline & \multirow{2}{*}{ RA } & \multirow{2}{*}{ Collision avoidance scheme } & \multirow{2}{*}{ Action time (second) } & \multicolumn{3}{|c|}{ SV } & \multirow{2}{*}{ Deviation } \\
\hline & & & & Before action & After action & Increment & \\
\hline \multirow{3}{*}{ Scenario I } & Aggressive & $U_{1}^{1}$ & 725 & 0.4 & 0.6 & 0.2 & 1 \\
\hline & Neutral & $U_{4}^{1}$ & 560 & 0.43 & 0.79 & 0.36 & 1.7 \\
\hline & Cautious & $U_{6}^{1}$ & 520 & 0.45 & 1 & 0.55 & 2 \\
\hline \multirow{3}{*}{ Scenario II } & Aggressive & $U_{1}^{2}$ & 845 & 1.05 & 1.2 & 0.15 & 0.9 \\
\hline & Neutral & $U_{3}^{\frac{1}{2}}$ & 835 & 1.05 & 1.25 & 0.2 & 1.8 \\
\hline & Cautious & $U_{6}^{3}$ & 0 & 1.6 & 2 & 0.4 & 5 \\
\hline
\end{tabular}

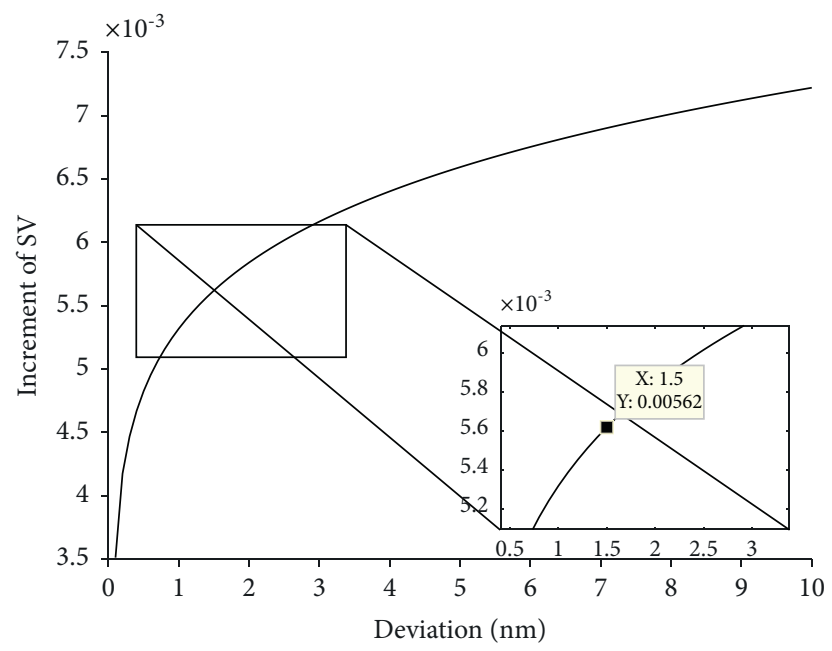

FIgURE 13: The fitness between the deviation and the increment of the SV.

(5) The relationships between cost (i.e., deviation) and profit (i.e., SV) are not linear. By simulating 100 cases of increasing the deviation from $0.1 \mathrm{~nm}$ to $10 \mathrm{~nm}$, the relationship between the deviation and the SV can be fitted with the following equation: 


$$
f(\text { Dev })=0.04739 * \operatorname{dev}^{1.13}+0.1079 .
$$

As shown in Figure 13, the fitness function indicates that before deviation less than $1.5 \mathrm{~nm}$, a slight avoidance action would increase the SV significantly, which is a good ratio of benefit. When the deviation is larger than $1.5 \mathrm{~nm}$, the ratio of benefit for ships that takes a large change is unsatisfactory.

\section{Conclusion and Future Research}

This study proposed a RA-CADM model to support collision avoidance decision making for MASS under man-machine hybrid conditions. On the basis of the collision risk model, the novelty of the model included the following: (1) it firstly applied the prospect theory in collision avoidance decision making and pioneered the MASS control with a human-like machine system; (2) the proposed model was tested with scenario simulations that covered the individual collision scenario and multiple collision scenarios to prove the reliability and the applicability in real cases; (3) the RA under different states were discussed in-depth, so as to present sufficient support for MASS designer and controller to make collision avoidance strategies. Although the results of this case study demonstrate risk preference, these risk parameters from other researches may deviate from the actual crews due to industry background differences.

Therefore, further research works are suggested as follows: (1) the differences between traditional CAS and intelligent CAS can be discussed; (2) the weight assignment for the RA indicators in the model should be investigated through surveys and or AIS data mining; (3) considering more risk-related factors during the decision-making process to optimize the collision schemes; and (4) real ship tests are encouraged for obtaining more information of collision avoidance decision.

\section{Data Availability}

The data used to support the findings of this study are available from the corresponding author upon request.

\section{Conflicts of Interest}

The authors declare that they have no conflicts of interest.

\section{Acknowledgments}

This work was supported by the National Natural Science Foundation of China (NSFC) under Grant No. 52031009.

\section{References}

[1] Z. Liu, Y. Zhang, X. Yu, and C. Yuan, "Unmanned surface vehicles: an overview of developments and challenges," $A n$ nual Reviews in Control, vol. 41, pp. 1-23, 2016.

[2] Y. Huang, L. Chen, P. Chen, R. R. Negenborn, and P. H. A. J. M. Van Gelder, "Ship collision avoidance methods: state-of-the-art," Safety Science, vol. 121, pp. 451-473, 2019.
[3] P. Koopman and M. Wagner, "Autonomous vehicle safety: an interdisciplinary challenge," IEEE Intelligent Transportation Systems Magazine, vol. 9, no. 1, pp. 90-96, 2017.

[4] L. P. Perera, J. P. Carvalho, and C. Guedes Soares, "Fuzzy logic based decision making system for collision avoidance of ocean navigation under critical collision conditions," Journal of Marine Science and Technology, vol. 16, no. 1, pp. 84-99, 2011.

[5] H. Lyu and Y. Yin, "COLREGS-constrained real-time path planning for autonomous ships using modified artificial potential fields," Journal of Navigation, vol. 72, no. 3, pp. 588-608, 2019.

[6] P. Chen, Y. Huang, J. Mou, and P. H. A. J. M. Van Gelder, "Probabilistic risk analysis for ship-ship collision: state-ofthe-art," Safety Science, vol. 117, pp. 108-122, 2018.

[7] Y. Huang, L. Chen, and P. H. A. J. M. Van Gelder, "Generalized velocity obstacle algorithm for preventing ship collisions at sea," Ocean Engineering, vol. 173, pp. 142-156, 2018.

[8] T. A. Johansen, T. Perez, and A. Cristofaro, "Ship collision avoidance and COLREGS compliance using simulation-based control behavior selection with predictive hazard assessment," IEEE Transactions on Intelligent Transportation Systems, vol. 17, no. 12, pp. 3407-3422, 2016.

[9] Y. Huang, L. Chen, R. R. Negenborn, and P. H. A. J. M. Van Gelder, "A ship collision avoidance system for human-machine cooperation during collision avoidance," Ocean Engineering, vol. 217, Article ID 107913, 2020.

[10] L. P. Perera, J. P. Carvalho, and C. Guedes Soares, "Intelligent ocean navigation and fuzzy-Bayesian decision/action formulation," IEEE Journal of Oceanic Engineering, vol. 37, no. 2, pp. 204-219, 2012.

[11] L. P. Perera, J. P. Carvalho, and C. G. Soares, "Solutions to the failures and limitations of Mamdani fuzzy inference in ship navigation," IEEE Transactions on Vehicular Technology, vol. 63, no. 4, pp. 1539-1554, 2013.

[12] Y. He, Y. Jin, L. Huang, Y. Xiong, P. Chen, and J. Mou, "Quantitative analysis of COLREG rules and seamanship for autonomous collision avoidance at open sea," Ocean Engineering, vol. 140, no. April, pp. 281-291, 2017.

[13] L. Li, G. Chen, and G. Li, "Study on auto decision-making and its simulation control for vessel collision avoidance," Proceedings of the 2015 Chinese Intelligent Automation Conference, vol. 338, pp. 265-275, 2015.

[14] K. Woerner, M. R. Benjamin, M. Novitzky, and J. J. Leonard, "Quantifying protocol evaluation for autonomous collision avoidance," Autonomous Robots, vol. 43, no. 4, pp. 967-991, 2019.

[15] Q. Yu, K. Liu, C.-H. Chang, and Z. Yang, "Realising advanced risk assessment of vessel traffic flows near offshore wind farms," Reliability Engineering \& System Safety, vol. 203, Article ID 107086, 2020.

[16] W. Naeem, G. W. Irwin, and A. Yang, "COLREGs-based collision avoidance strategies for unmanned surface vehicles," Mechatronics, vol. 22, no. 6, pp. 669-678, 2012.

[17] M.-C. Fang, K.-Y. Tsai, and C.-C. Fang, "A simplified simulation model of ship navigation for safety and collision avoidance in heavy traffic areas," Journal of Navigation, vol. 71, no. 4, pp. 837-860, 2018.

[18] Q. Yu, K. Liu, Z. Yang, H. Wang, and Z. Yang, "Geometrical risk evaluation of the collisions between ships and offshore installations using rule-based Bayesian reasoning," Reliability Engineering \& System Safety, vol. 210, Article ID 107474, 2021.

[19] H. Shen, H. Hashimoto, A. Matsuda, Y. Taniguchi, D. Terada, and C. Guo, "Automatic collision avoidance of multiple ships 
based on deep Q-learning," Applied Ocean Research, vol. 86, pp. 268-288, 2018.

[20] L. Hu, W. Naeem, E. Rajabally et al., "A multiobjective optimization approach for COLREGs-Compliant path planning of autonomous surface vehicles verified on networked bridge simulators," IEEE Transactions on Intelligent Transportation Systems, vol. 21, no. 3, pp. 1167-1179, 2020.

[21] Y. Zheng, X. Zhang, Z. Shang, S. Guo, and Y. Du, "A decisionmaking method for ship collision avoidance based on improved cultural particle swarm," Journal of Advanced Transportation, vol. 2021, Article ID 8898507, 31 pages, 2021.

[22] S. Li, J. Liu, and R. R. Negenborn, "Distributed coordination for collision avoidance of multiple ships considering ship maneuverability," Ocean Engineering, vol. 181, no. 1178, pp. 212-226, 2019.

[23] D. Kim, K. Hirayama, and T. Okimoto, "Distributed stochastic search algorithm for multi-ship encounter situations," Journal of Navigation, vol. 70, no. 4, pp. 699-718, 2017.

[24] J. Zhang, D. Zhang, X. Yan, S. Haugen, and C. Guedes Soares, "A distributed anti-collision decision support formulation in multi-ship encounter situations under COLREGs," Ocean Engineering, vol. 105, pp. 336-348, 2015.

[25] R. Szlapczynski, "Evolutionary sets of safe ship trajectories within traffic separation schemes," Journal of Navigation, vol. 66, no. 1, pp. 65-81, 2013.

[26] X. Chen, Z. Li, Y. Yang, L. Qi, and R. Ke, "High-resolution vehicle trajectory extraction and denoising from aerial videos," IEEE Transactions on Intelligent Transportation Systems, vol. 22, no. 5, pp. 3190-3202, 2020.

[27] M. Liang, R. W. Liu, S. Li, Z. Xiao, X. Liu, and F. Lu, “An unsupervised learning method with convolutional auto-encoder for vessel trajectory similarity computation," Ocean Engineering, vol. 225, Article ID 108803, 2021.

[28] Q. Yu, K. Liu, A. P. Teixeira, and C. G. Soares, "Assessment of the influence of offshore wind farms on ship traffic flow based on AIS data," Journal of Navigation, vol. 73, no. 1, pp. 131-148, 2020.

[29] W. Zhang, F. Goerlandt, J. Montewka, and P. Kujala, “A method for detecting possible near miss ship collisions from AIS data," Ocean Engineering, vol. 107, pp. 60-69, 2015.

[30] Y. Wen, Y. Huang, C. Zhou, J. Yang, C. Xiao, and X. Wu, "Modelling of marine traffic flow complexity," Ocean Engineering, vol. 104, pp. 500-510, 2015.

[31] Z. Cheng, Y. Li, and B. Wu, "Early warning method and model of inland ship collision risk based on coordinated collisionavoidance actions," Journal of Advanced Transportation, vol. 2020, Article ID 5271794, 14 pages, 2020.

[32] R. Szlapczynski and J. Szlapczynska, "Review of ship safety domains: models and applications," Ocean Engineering, vol. 145, pp. 277-289, 2017.

[33] Y. Huang and P. H. A. J. M. Van Gelder, "Time-varying risk measurement for ship collision prevention," Risk Analysis, vol. 40, no. 1, pp. 24-42, 2020.

[34] J. Park, M. Han, and W. Baek, "Quantifying the performance impact of large pages on in-memory big-data workloads," in Proceeding 2016 IEEE International Symposium on Workload Characterization IISWC, Providence, RI, US, May 2016.

[35] J. Ma, W. Li, C. Jia, C. Zhang, and Y. Zhang, "Risk prediction for ship encounter situation awareness using long short-term memory based deep learning on intership behaviors," Journal of Advanced Transportation, vol. 2020, Article ID 8897700, 15 pages, 2020.
[36] B. Lin, "Behavior of ship officers in maneuvering to prevent a collision," Journal of Marine Science and Technology, vol. 14, no. 4, pp. 225-230, 2006.

[37] J.-B. Yim, D.-S. Kim, and D.-J. Park, "Modeling perceived collision risk in vessel encounter situations," Ocean Engineering, vol. 166, pp. 64-75, 2018.

[38] C. D. Wickens, A. Williams, B. A. Clegg, and C. A. P. Smith, "Nautical collision avoidance," Human Factors: The Journal of the Human Factors and Ergonomics Society, vol. 62, no. 8, pp. 1304-1321, 2020.

[39] T. Aven, "On the meaning and use of the risk appetite concept," Risk Analysis, vol. 33, no. 3, pp. 462-468, 2013.

[40] D. Kai-Ineman and A. Tversky, "Prospect theory: an analysis of decision under risk," Econometrica, vol. 47, no. 2, pp. 363-391, 1979.

[41] A. Tversky and D. Kahneman, "Advances in prospect theory: cumulative representation of uncertainty," Journal of Risk and Uncertainty, vol. 5, no. 4, pp. 297-323, 1992.

[42] U. Schmidt, C. Starmer, and R. Sugden, "Third-generation prospect theory," Journal of Risk and Uncertainty, vol. 36, no. 3, pp. 203-223, 2008.

[43] L. Wang, Y.-M. Wang, and L. Martínez, "A group decision method based on prospect theory for emergency situations," Information Sciences, vol. 418-419, pp. 119-135, 2017.

[44] S. Gao, E. Frejinger, and M. Ben-Akiva, "Adaptive route choices in risky traffic networks: a prospect theory approach," Transportation Research Part C: Emerging Technologies, vol. 18 , no. 5, pp. 727-740, 2010.

[45] K. Hjorth and M. Fosgerau, "Using prospect theory to investigate the low marginal value of travel time for small time changes," Transportation Research Part B: Methodological, vol. 46, no. 8, pp. 917-932, 2012.

[46] P. Liu, F. Jin, X. Zhang, Y. Su, and M. Wang, "Research on the multi-attribute decision-making under risk with interval probability based on prospect theory and the uncertain linguistic variables," Knowledge-Based Systems, vol. 24, no. 4, pp. 554-561, 2011.

[47] L. Zhou, S. Zhong, S. Ma, and N. Jia, "Prospect theory based estimation of drivers' risk attitudes in route choice behaviors," Accident Analysis \& Prevention, vol. 73, pp. 1-11, 2014.

[48] R. W. Liu, J. Nie, S. Garg, Z. Xiong, Y. Zhang, and M. S. Hossain, "Data-driven trajectory quality improvement for promoting intelligent vessel traffic services in 6G-enabled maritime IoT systems," IEEE Internet Things Journal, vol. 8, no. 7, pp. 5374-5385, 2020.

[49] X. Chen, L. Qi, Y. Yang et al., "Video-based detection infrastructure enhancement for automated ship recognition and behavior analysis," Journal of Advanced Transportation, vol. 2020, Article ID 7194342, 12 pages, 2020.

[50] J. Li, H. Wang, Z. Guan, and C. Pan, "Distributed multiobjective algorithm for preventing multi-ship collisions at sea," Journal of Navigation, vol. 73, no. 5, pp. 971-990, 2020.

[51] Y. Hu, A. Zhang, W. Tian, J. Zhang, and Z. Hou, "Multi-ship collision avoidance decision-making based on collision risk index," Journal of Marine Science and Engineering, vol. 8, no. 9 , p. $640,2020$.

[52] K. Zhou, J. Chen, and X. Liu, "Optimal collision-avoidance manoeuvres to minimise bunker consumption under the twoship crossing situation," Journal of Navigation, vol. 71, no. 1, pp. 151-168, 2018.

[53] L. Hu, W. Naeem, E. Rajabally et al., "COLREGs-compliant path planning for autonomous surface vehicles: a multiobjective optimization approach $* *$ the authors should like to thank innovate UK, grant reference, tsb 102308, for the 
funding of this project," IFAC-PapersOnLine, vol. 50, no. 1, pp. 13662-13667, 2017.

[54] D. Kahneman and A. Tversky, "Prospect theory: an analysis of decision under risk," Econometrica, vol. 47, no. 2, pp. 263-292, 1979.

[55] H. Xu, J. Zhou, and W. Xu, "A decision-making rule for modeling travelers' route choice behavior based on cumulative prospect theory," Transportation Research Part C: Emerging Technologies, vol. 19, no. 2, pp. 218-228, 2011.

[56] Z. Jian-min, "An experimental test on cumulative prospect theory," University Natural Sciences Education, vol. 1, 2007. 